$\begin{array}{cl}\begin{array}{c}\text { Revue } \\ \text { de I'histoire }\end{array} & \text { Revue de l'histoire des religions } \\ \text { des religions } & \begin{array}{l}1 \mid 2014 \\ \text { Varia }\end{array}\end{array}$

\title{
Journalier de Jean Pussot, maître-charpentier à Reims
} (1568-1626)

Présentation, édition et notes par Stefano Simiz et Jérôme Buridant, préface de Patrick Demouy, Presses universitaires du Septentrion (« Documents et témoignages », série Histoire), Villeneuve d'Ascq, 2008

\section{Albrecht Burkardt}

\section{(2) OpenEdition Journals}

\section{Édition électronique}

URL : https://journals.openedition.org/rhr/8221

DOI : 10.4000/rhr.8221

ISSN : 2105-2573

\section{Éditeur}

Armand Colin

Édition imprimée

Date de publication : 1 mars 2014

Pagination : 145-148

ISBN : 978-2200929107

ISSN : 0035-1423

\section{Référence électronique}

Albrecht Burkardt, « Journalier de Jean Pussot, maître-charpentier à Reims (1568-1626) », Revue de I'histoire des religions [En ligne], 1 | 2014, mis en ligne le 13 mai 2014, consulté le 21 septembre 2021. URL : http://journals.openedition.org/rhr/8221; DOI : https://doi.org/10.4000/rhr.8221

Ce document a été généré automatiquement le 21 septembre 2021.

Tous droits réservés 


\section{Journalier de Jean Pussot, maître- charpentier à Reims (1568-1626)}

Présentation, édition et notes par Stefano Simiz et Jérôme Buridant, préface de Patrick Demouy, Presses universitaires du Septentrion (« Documents et témoignages », série Histoire), Villeneuve d'Ascq, 2008

\section{Albrecht Burkardt}

\section{RÉFÉRENCE}

Journalier de Jean Pussot, maître-charpentier à Reims (1568-1626). Présentation, édition et notes par Stefano Simiz et Jérôme Buridant, préface de Patrick Demouy, Presses universitaires du Septentrion (« Documents et témoignages ", série Histoire), Villeneuve d'Ascq, 2008, 290 p., 24 cm, $22 €$, ISBN 978-2-85939-999-3.

1 Cette édition du Journalier de Jean Pussot met à la disposition des lecteurs un « égodocument» assez détaillé de la période qui va des guerres de Religion au règne de Louis XIII. Sans être méconnu des spécialistes, il restait difficile d'accès : la première publication, dans les Travaux de l'Académie Impériale de Reims, date des années 1850. Il est vrai que le manuscrit de ce maître-artisan rémois ne saurait concurrencer, en quantité tout au moins, la masse documentaire que fournissent certains textes analogues de l'époque, comme les Mémoires de Claude Haton, voire les Mémoires-Journaux d'un Pierre de l'Estoile. Cependant, pour l'histoire rémoise, ce texte constitue un document sans doute unique qui, s'il comporte la description d'événements dont l'importance dépasse largement le cadre de la ville (on y trouve par exemple une description détaillée du sacre de 1610), était déjà estimé des contemporains pour sa précision sur le plan local. Sous l'œil de l'historien, le texte s'affirme encore comme une source fort suggestive : selon les éditeurs, il permet de se faire une image assez fiable et différenciée des décennies concernées, autant $\mathrm{du}$ point de vue socio-économique que dans une perspective politico-religieuse. Il ne faut pas pour autant s'attendre à un tableau exhaustif: les données ne manquent pas qui se trouvent omises dans le Journalier, ou 
étonnamment peu représentées; ainsi des évolutions au sein de l'élite urbaine, mais aussi de la vie professionnelle de l'auteur. On ne sort pas en effet avec ce texte des lois du genre, dont une écriture quelque peu « impressionniste » fait partie intégrante.

Dans ce cadre, le Journalier connaît une évolution assez nette. Si les premières années, voire les premières décennies, sont marquées par une écriture assez proche du style d'un livre de raison "classique » - dans un ensemble d'énoncés assez peu loquace, les informations sur la vie familiale prennent la place proportionnellement la plus importante -, plus on avance dans le temps plus le texte gagne en extensivité. Chemin faisant, les priorités thématiques se modifient - à ce point que l'auteur, à la fin du journal, et de sa vie, cherche à "rattraper » un certain nombre de données qui, au moment qu'aurait voulu la chronologie, n'étaient alors visiblement pas entrées dans ses priorités. Jamais totalement absents du texte, les événements politiques atteignent leur plus grande présence dans les années 1588-1597, avec la fin des guerres de Religion et l'avènement d'Henri IV. Par la suite, la priorité revient aux questions de pratique religieuse - tout à fait en parallèle, cette fois, avec l'emprise de la Contre-Réforme sur le pays. Sans variations dans le temps : les questions de "conjonctures", économique et météorologique, qui gardent une importance certaine tout au long du Journalier. Les informations sont précises au point que l'évolution des prix se dessine nettement pour toute la période en question.

3 Comme toute personne posant, par le moyen de l'écriture, en témoin de son temps, Pussot fait exception à son milieu dans la mesure même où il s'en veut le représentant. La façon de se caractériser lui-même - «ne suis ni grand philosophe, orateur, rhétoricien, légiste et praticien, mais humble et simple charpentier en la ville de Reims »- le manifeste à sa façon: la modestie affichée est sans doute sincère et pourtant une litote. Cela dit, Pussot ne saurait échapper aux empreintes du monde qui est le sien. C'est vrai pour ses orientations "économiques"-ses observations continues des intempéries, de la pluie et du beau temps sont certes des plus courantes dans ce "siècle de fer", mais correspondent aussi à sa passion pour la culture des vignes : comme c'est l'habitude des bonnes familles de la bourgeoisie moyenne et élevée rémoise, le maître charpentier possède une propriété viticole dans les campagnes aux alentours de la ville. C'est vrai également pour les pratiques culturelles. Malgré la familiarité avec l'écrit, les lectures de Pussot ne semblent guère avoir dépassé un nombre assez restreint; son goût de l'écriture va de pair avec celui pour les rimes, l'inspiration lui «provenant le plus souvent des chants, proverbes ou dictons populaires » selon les éditeurs. Par ailleurs, l'auteur est aussi un chroniqueur constant des faits de guerre, et là encore l'optique du « quotidien » l'emporte, les événements rapportés reflétant la « violence ordinaire » bien davantage que les hauts faits d'armes. Ce sont les convictions religieuses de l'auteur qui paraissent les plus originales; et encore les passions du temps ont laissé leurs marques évidentes. Pendant les guerres de Religion, Pussot est le représentant d'un catholicisme radicalisé qui ne cache pas ses sympathies pour la Ligue. Il devient plus modéré par la suite, avec la conversion d'Henri IV. Ses connaissances du protestantisme sont assez réduites; en revanche, l'auteur a bien des sympathies pour les positions d'un Érasme. Il reste très sceptique quant aux « superstitions » du temps, à commencer par la sorcellerie (sa persécution incluse !) ; il se méfie des attitudes bigotes et faussement mystiques. C'est le partisan d'une sorte de religion civique dont le centre d'activité est la vie paroissiale, avec comme idéal une « dévotion bien réglée ». Aussi, même s'il garde une certaine méfiance 
vis-à-vis des ordres religieux, il partage en une large mesure les préoccupations de la Réforme catholique - du moins en ce qui concerne la dénonciation des abus de toutes sortes. Plus généralement, Pussot est fortement hostile à tout ce qui est ambition et apparences, chez les clercs autant que chez les laïcs, ce qui n'empêche pas qu'il donne beaucoup d'attention aux pratiques religieuses dans leurs aspects liturgiques, et qu'il a une grande admiration pour les fêtes de l'Église dans leur éclat baroque. Avec les années, en effet, Pussot a gagné une estime certaine pour la religion dans ses fonctions stabilisatrices au sein de l'État - ce qui va de pair avec une révérence croissante pour l'institution royale, perdue temporairement, suite à la « trahison » d'Henri III.

4 L'ensemble de ces traits se trouve exposé de façon convaincante dans la longue introduction (p.15-75) au Journalier (le lecteur aura cependant quelque peine à retrouver les passages cités, car les références aux pages dans l'édition qui suit ne sont pas indiquées). Quant au fond, la seule chose que l'on puisse regretter est le peu de comparaisons effectuées dans ce texte de présentation avec d'autres sources contemporaines de type analogue en France, mais aussi avec celles étudiées dans d'autres pays, lesquelles auraient permis d'inscrire cette publication dans l'actualité de la recherche. Quelques remarques critiques s'imposent également à propos de l'édition du Journalier. Ses modalités ne sont pas expliquées avec précision, et ce qu'elle propose entre parfois en contradiction avec les assertions de l'introduction: la mise en forme du texte comprend, par exemple, l'élimination des ratures, alors que les éditeurs montrent très bien à quel point l'étude de ces dernières peut enrichir sa compréhension. En d'autres termes, les auteurs n'ont pas voulu établir une véritable édition critique, et le souci de ne pas trop alourdir le texte se traduit également par un nombre assez restreint d'annotations. Au sens strict, il ne s'agit d'ailleurs que d'une édition partielle : le manuscrit original conservé à la bibliothèque municipale de Reims (manuscrit dont la description reste quelque peu opaque) comporte également les comptes établis par Pussot, non repris ici et peu commentés par les éditeurs. Ces remarques, en somme, n'amoindrissent pas le mérite des éditeurs qu'il faut remercier d'avoir rendu de nouveau accessible le Journalier qu'un plan de Reims et un glossaire aident à rendre plus maniable, au même titre que des index tout à fait utiles.

\section{AUTEUR}

\section{ALBRECHT BURKARDT}

Université de Limoges 